\title{
APRENDIZAGEM ATIVA COMO PRINCIPAL ESTRATÉGIA PARA ATENDIMENTO DAS NOVAS DIRETRIZES CURRICULARES NACIONAIS EM ENGENHARIA
}

Elio Molisani Ferreira Santos 1,2 - molisani@usp.br

Lucas Ribeiro Mata ${ }^{1}$-lucasmata@usp.br

Fillipe Rocha Esteves ${ }^{1}$ - fillipe.esteves@usp.br

Roseli de Deus Lopes ${ }^{1}$ - roseli.lopes@usp.br

${ }^{1}$ Universidade de São Paulo, Escola Politécnica, Programa de Pós-Graduação em Engenharia Elétrica (PPGEE)

Av. Professor Luciano Gualberto, travessa 3, 158

CEP 05508-010 - São Paulo - SP

${ }^{2}$ Universidade Federal do Amazonas, Instituto de Ciências Exatas, Departamento de Física Av. Rodrigo Otávio, $n^{o}$ 6.200, Campus Universitário Senador Arthur Virgílio Filho, Setor Norte, Coroado I

CEP 69077- 000 - Manaus - AM

Resumo: O ensino de engenharia no Brasil enfrenta grandes desafios diante da transformação digital, da economia global e da chamada "universalização do conhecimento". As diretrizes curriculares têm sido aprimoradas para que possibilite a formação de profissionais cada vez mais capazes de promover o desenvolvimento de tecnologias, inovação e conhecimento, tarefas intimamente ligadas à profissão do engenheiro. Neste contexto, ampla discussão tem sido realizada acerca dos métodos de aprendizagem ativa, os quais visam trazer o aluno para o centro do processo de aprendizagem, motivando-lhe a buscar por conta própria o conhecimento. Para que isto ocorra, são necessárias ações institucionais nas universidades e mudança na compreensão dos papéis do professor e do aluno, para que juntos sejam capazes de potencializar uma aprendizagem mais estruturadora. Este trabalho apresenta uma revisão acerca da aprendizagem ativa em cursos de engenharia à luz das novas Diretrizes Curriculares Nacionais do Curso de Graduação em Engenharia. Também se discute a eficácia destas estratégias frente aos desafios atuais da educação em engenharia e suas vantagens $e$ desvantagens em comparação ao método de ensino tradicional. Por fim, dois casos práticos de aplicação da aprendizagem ativa em cursos de engenharia são analisados e discutidos.

Palavras-chave: Aprendizagem Ativa. Ensino de Engenharia. Diretrizes Curriculares Nacionais de Engenharia. Pirâmide da Aprendizagem. 


\section{INTRODUÇÃO}

As situações que o engenheiro deve enfrentar no ambiente profissional requerem habilidades que extrapolam a mera execução de normas pré-estabelecidas. $O$ avanço extremamente veloz do conhecimento e de sua universalização, graças aos meios de comunicação digital, processo conhecido como "descentralização da inteligência" (SILVEIRA, 2005), implica na capacidade do engenheiro de buscar novas e melhores formas de interagir em seu ambiente profissional e, idealmente, criar novos caminhos para atingir mais eficientemente os resultados esperados.

O preparo dos alunos de engenharia para esta nova realidade deve contemplar o desenvolvimento de habilidades para a solução problemas reais, com a versatilidade para vir a trabalhar com problemas ainda desconhecidos. O engenheiro de hoje deve, principalmente, aprender a aprender. Seu sucesso formativo, está atrelado a uma aprendizagem significativa, que envolve a aprendizagem de conceitos e, também, desenvolve habilidades diversas e complexas, atitudes e valores, conjunto este denominado por competências.

Atualmente, alunos e professores são desafiados a repensarem seus papéis no processo ensino-aprendizagem. Subjetivamente, o professor deve estar preparado para promover situações em que o estudante seja motivado a buscar e interpretar informações e a trabalhar em equipe para resolver problemas reais. Deve ser um mediador atento para criar ferramentas e ambientes favoráveis ao desenvolvimento cognitivo, de habilidades conceituais, atitudinais e procedimentais, à níveis mais avançados como análise, síntese e criação (ANDERSON et al., 2001). Aos estudantes, cabe uma nova postura diante do processo educacional, devem abandonar o hábito de compreender apenas as partes, visando somente aprovação em testes, e passar a vislumbrar o todo para uma compreensão ampla e profunda dos fenômenos.

As características apresentadas até agora como uma "nova realidade" do ensino de engenharia convergem para o chamado "método ativo de aprendizagem", que tem recebido atenção dos educadores como possíveis respostas às demandas educacionais e profissionais atuais. Neste contexto, o objetivo do presente trabalho é apresentar uma breve revisão acerca da aprendizagem ativa em cursos de engenharia à luz das novas Diretrizes Curriculares Nacionais do Curso de Graduação em Engenharia, destacando sua importância estratégica ao desenvolvimento do país e relatos de estudantes que vivenciaram sua aplicação.

\subsection{Capital humano: um desafio para o Brasil}

Nos últimos anos, o Brasil tem enfrentado um grande desafio relacionado a competitividade de sua economia no mercado global. No Índice Global de Inovação (IGI), indicador elaborado conjuntamente pela Universidade de Cornell, INSEAD e Organização Mundial da Propriedade Intelectual, o país encontra-se abaixo da média mundial, ocupando a $66^{\mathrm{a}}$ posição na lista dos 128 países analisados. Investigando as variáveis utilizadas no cálculo do IGI, constata-se que o Brasil apresentou fraco desempenho nos indicadores relacionados aos recursos humanos (WIPO, 2019).

O Fórum Econômico Mundial publicou, em 2018, o relatório Readiness for the future production para avaliar o posicionamento de cada uma das 100 maiores economias na corrida tecnológica da indústria 4.0. As variáveis consideradas na avaliação foram agrupadas em dois indicadores: vetores de produção e estrutura produtiva. $\mathrm{O}$ indicador vetores de produção é calculado com base em seis aspectos: tecnologia e inovação, capital humano, comércio global e investimento, estrutura institucional, recursos naturais e demanda. A estrutura produtiva compreende a complexidade e escala da produção do país analisado. Segundo relatório, o Brasil e demais países da América Latina foram incluídos no grupo de países classificados como nascentes, sendo caracterizados por base produtiva fraca e baixo nível de preparo para o 
futuro da produção (WEF, 2018). No ranking geral, o Brasil ocupa a $47^{\mathrm{a}}$ posição no indicador vetores de produção e, no indicador estrutura produtiva, ocupa a $41^{\mathrm{a}}$ posição. $\mathrm{O}$ pior desempenho do Brasil foi observado na variável capital humano, levando o país a ocupar a $74^{\circ}$ posição no ranking do capital humano.

Os dados apresentados pelos dois relatórios analisados apontam que uma das questões mais críticas para o Brasil, neste momento de profundas transformações, é o seu capital humano. A busca por avanços na formação e qualificação dos recursos humanos do país é imprescindível para melhoria do posicionamento da economia brasileira, em termos de produtividade e competitividade, no mercado global.

\subsection{Novas diretrizes para cursos de engenharia}

Tendo em vista os desafios do Brasil diante da transformação digital da economia global, o Ministério da Educação (MEC), por meio da Câmara de Educação Superior (CES) do Conselho Nacional de Educação (CNE), homologou a Resolução CNE/CES No 2 de 24 de abril de 2019 que instituiu novas Diretrizes Curriculares Nacionais do Curso de Graduação em Engenharia (DCNs de Engenharia).

As diretrizes curriculares definem as competências - conjunto de conhecimentos, habilidades, atitudes e valores - que devem ser desenvolvidas ao longo do curso, estabelecendo também normas para organização, desenvolvimento e avaliação dos cursos de engenharia. As Instituições de Ensino Superior (IES) possuem autonomia e flexibilidade na elaboração do currículo e plano pedagógico do curso.

Dentre as inovações trazidas pelas novas DCNs, destaca-se o estabelecimento de capítulos com definições mais claras acerca dos agentes estruturantes dos cursos de engenharia, como são os casos do "Capítulo II - Do Perfil e Competências Esperadas do Egresso" e do "Capítulo III - Da Organização do Curso de Graduação em Engenharia", que abordam de forma mais específica as características desejáveis na formação dos graduandos e no desenvolvimento das atividades do curso.

No relatório do processo de elaboração das novas DCNs é salientada a importância da adoção de metodologias de ensino baseadas na aprendizagem ativa desde o primeiro ano do curso, promovendo uma educação mais centrada no aluno e preocupada com o desenvolvimento de competências. Dentro da proposta de modernização dos cursos engenharia, o relatório aponta também a necessidade de uma maior integração empresa-escola e valorização da inter e transdisciplinaridade (MEC, 2020).

\section{APRENDIZAGEM ATIVA NO ENSINO DE ENGENHARIA}

A aprendizagem ativa pode ser entendida como como o planejamento de um conjunto de ações estratégicas educacionais que incentivem os participantes a interagir, refletir, aplicar e compartilhar suas experiências (INSTRUCTION, 2010). Em outras palavras, a ideia é transformar o aluno no agente principal do processo educacional, motivando sua busca consciente por um conhecimento que lhe seja significativo.

No entanto, a receptividade dos estudantes às abordagens de aprendizagem ativa esbarra no desafio da mudança de atitude frente ao compromisso com a aprendizagem. Uma vez vencida esta etapa, os alunos passam a encarar com mais naturalidade tais abordagens. Por parte dos docentes, na maioria das vezes formados por uma escola tradicional, a receptividade é variada. Existem muitos professores e cursos que já utilizam estratégias de aprendizagem ativa, tendo ou não plena consciência disto. A contextualização de conteúdos, discussões e até trabalhos interdisciplinares têm se tornado mais comuns em sala de aula. A dificuldade, 
entretanto, reside na crença de que a cobertura de todo o conteúdo por parte do professor exprime melhor aproveitamento em uma disciplina.

Tal dificuldade reforça a existência de realidades diametralmente opostas dentro das estratégias de aprendizado. O professor, enquanto mediador do aprendizado dos alunos, deve fazer a dura opção entre a quantidade de conteúdo e aquilo que é estruturante para o desenvolvimento de determinadas competências. Esta mudança de paradigma delineia um processo de aprendizagem, principalmente no exercício da docência. É preciso compreender que o objetivo do trabalho da escola deve, para além do conhecimento conceitual e técnico, privilegiar o desenvolvimento de autonomia, visão crítica, capacidade de modelagem, representação e comunicação.

Neste contexto, os procedimentos para a verificação da aprendizagem também requerem revisões. Tornou-se mais palatável a adoção de relatórios, entrevistas, artigos científicos, avaliações orais e projeto anual como forma de avaliação. Também costuma-se pesquisar a inserção no mercado de trabalho, como indicador de sucesso do método utilizado.

A fim de motivar a curiosidade e a organização dos estudantes, é comum propor-se um problema inicial, como um material potencializador, a partir do qual o conhecimento vai sendo construído. As estratégias baseadas em problemas procuram tal apropriação do conhecimento a partir da assimilação e acomodação, fazendo com que o aluno seja exposto a certa interação entre a teoria estudada e a vida real. Desta forma a aprendizagem torna-se uma relação de interação entre o sujeito e o objeto de estudo. Como muitos dos problemas reais são essencialmente complexos, tal interação também passa a se tornar multidisciplinar.

Outra estratégia de grande valor para o ensino de engenharia é a aprendizagem baseada em projetos, onde os estudantes, reunidos em grupos, desenvolvem um projeto a partir de um problema, necessidade ou observação de algum fenômeno. O professor assume o papel de orientador, acompanhando e supervisionando o desenvolvimento dos projetos.

\subsection{Aprendizagem ativa versus ensino tradicional}

A aprendizagem ativa não é um assunto novo (MORÁN, 2015), mas boa parte dos educadores não se sente confortável em adotar tal prática muitas vezes pela falta de clareza quanto às suas características, seus benefícios ou pela dificuldade em empregar essa metodologia (GONÇALVES; SCAICO, 2019), dando preferência ao ensino tradicional, que continua sendo fortemente verificado no cotidiano educacional (MARIN et al., 2010).

O modelo tradicional de ensino, predominantemente adotado nos séculos XIX e XX (CASTANHA, 2017; SAVIANI et al., 2017), é caracterizado especialmente pela atuação do professor como transmissor de conhecimento. Por parte dos alunos destaca-se a capacidade de memorização e a habilidade para a resolução de exercícios descontextualizados por técnicas exaustivas de repetição, puramente mecânicas, que pouco levam a uma compreensão mais profunda acerca dos conceitos transmitidos ou da aplicação prática dos mesmos. Este tipo de ensino, também chamado de ensino bancário por Paulo Freire, onde o professor deposita o conhecimento e para depois o sacá-lo através de provas, dificilmente oferece oportunidades de reflexões mais profundas e complexas acerca do mundo em que se vive (FREIRE, 1987).

Para facilitar a visualização das principais diferenças entre a aprendizagem ativa e o ensino tradicional, é apresentado na Tabela 1 um quadro resumindo algumas de suas características (HARKINS, 2008; MORÁN, 2017; BERBEL, 2011). 
Tabela 1 - Comparação entre Aprendizagem Ativa e Ensino Tradicional.

\begin{tabular}{|c|c|c|}
\hline $\begin{array}{c}\text { Atuação dos } \\
\text { personagens do } \\
\text { processo educacional }\end{array}$ & Aprendizagem Ativa & Ensino Tradicional \\
\hline O professor é... & Mediador no processo de aprendizagem. & A fonte e o transmissor do conhecimento. \\
\hline $\begin{array}{l}\text { A atuação do professor } \\
\text { é... }\end{array}$ & De parceria para promover autonomia. & Controladora, favorecendo a obediência. \\
\hline O aluno é... & $\begin{array}{l}\text { O agente principal do processo de } \\
\text { aprendizagem. }\end{array}$ & $\begin{array}{l}\text { Dependente das instruções do professor } \\
\text { adquirir conhecimento. }\end{array}$ \\
\hline A atuação do aluno é & $\begin{array}{l}\text { Dinâmica, participa ativamente do } \\
\text { processo de aprendizagem. }\end{array}$ & $\begin{array}{l}\text { Passiva, recebe o conteúdo nas aulas } \\
\text { expositivas e executa testes e provas. }\end{array}$ \\
\hline O conhecimento é... & $\begin{array}{l}\text { Socialmente construído, relacionando } \\
\text { teoria e prática, através de fontes } \\
\text { colaborativas e diversas de informação. }\end{array}$ & $\begin{array}{l}\text { Transmitido pelo professor e por livros } \\
\text { didáticos. }\end{array}$ \\
\hline A tecnologia digital é... & $\begin{array}{l}\text { Uma ferramenta poderosa que pode ser } \\
\text { usada pelo aluno para buscar e } \\
\text { compartilhar informação, simular, etc. }\end{array}$ & $\begin{array}{l}\text { Usada apenas pelo professor para transmitir } \\
\text { informação por meio de apresentações, } \\
\text { vídeos, etc. }\end{array}$ \\
\hline O material didático é... & $\begin{array}{l}\text { variado, contendo elementos analógicos } \\
\text { e digitais com múltiplas linguagens. }\end{array}$ & $\begin{array}{l}\text { Composto principalmente por cadernos e } \\
\text { livros. }\end{array}$ \\
\hline $\begin{array}{l}\text { O processo de avaliação } \\
\text { é... }\end{array}$ & $\begin{array}{l}\text { Contínuo, com acompanhamento } \\
\text { constante por parte do professor. }\end{array}$ & $\begin{array}{l}\text { intervalado, geralmente ao término de um } \\
\text { bloco de conteúdos. }\end{array}$ \\
\hline $\begin{array}{l}\text { Os instrumentos de } \\
\text { avaliação são... }\end{array}$ & $\begin{array}{l}\text { Diversificados, visando analisar o } \\
\text { desenvolvimento de inúmeras } \\
\text { competências (conhecimento, } \\
\text { habilidades, atitudes e valores). }\end{array}$ & $\begin{array}{l}\text { Similares, para verificar a aquisição de } \\
\text { conhecimentos específicos através de provas } \\
\text { individuais escritas ou orais, sem consulta a } \\
\text { materiais de referência. }\end{array}$ \\
\hline
\end{tabular}

Fonte: Autoria própria.

Vale ressaltar aqui um diagrama amplamente divulgado na internet que visa enaltecer os benefícios da aprendizagem ativa em comparação com o ensino tradicional. Este diagrama, ilustrado na Figura 1, denominado cone ou pirâmide da aprendizagem, que é atrelado aos percentuais de sucesso ou insucesso das práticas educacionais adotadas em sala de aula é erroneamente atribuído a uma publicação de Edgar Dale datada de 1946 ou a um suposto modelo citado pelo médico psiquiatra William Glasser na década de 1960. Estes valores percentuais foram posteriormente combinados ao diagrama, sem a existência de qualquer evidência científica criteriosa que comprove tais números (DA SILVA; MUZARDO, 2018).

Ainda assim, o cone da experiência - como era denominado por Edgar Dale (DALE, 1954) - se analisado de forma coerente, é muito valioso para que se perceba o alcance de cada prática metodológica no meio educacional e, então, conscientemente seja sustentada a adoção de práticas pedagógicas que visam enaltecer a participação mais ativa do estudante.

Na Figura 2 pode ser observada uma representação da primeira versão do cone da experiência de Dale (ACLAND, 2017), adaptada e renomeada aqui como cone de aprendizagem. Os elementos internos à figura do cone trazem as informações originais propostas por Edgar Dale, e todos os elementos externos à área do cone são adequações realizadas por esse grupo de autores. 
Figura 1 - Cone ou pirâmide da aprendizagem erroneamente atribuído a Edgar Dale.

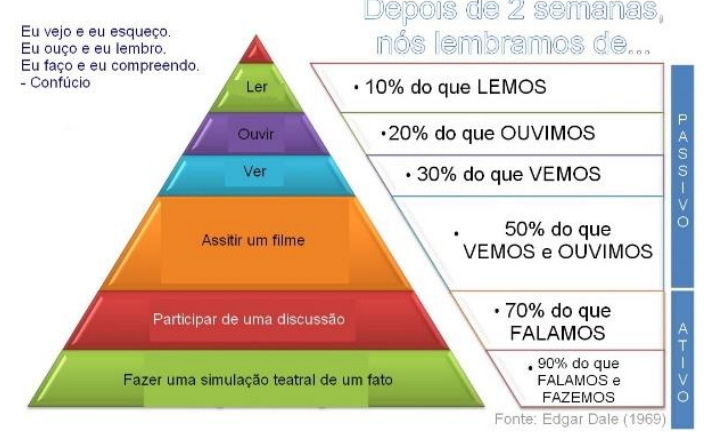

Fonte: Imagem retirada de pesquisa no Google.

Disponível em http://binfoutil.blogspot.com/2012/07/piramide-do-aprendizado.html. Acesso em 24 jul. 2020.

Figura 2 - Cone da Aprendizagem: adaptação do cone da experiência de E. Dale.

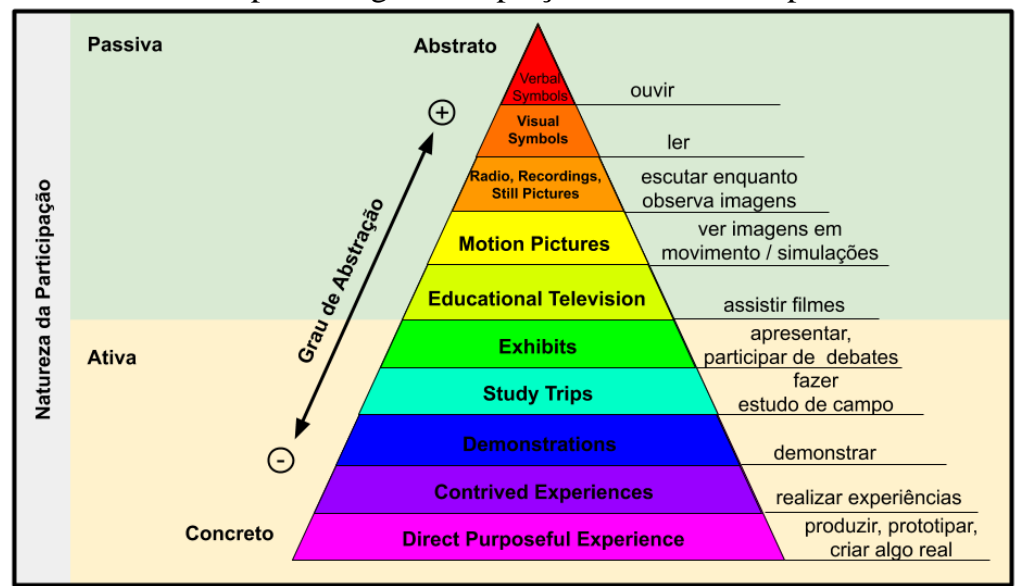

Fonte: Autoria própria, com base no primeiro cone da experiência proposto por Edgar Dale em 1946.

O arranjo do cone não está baseado em nível de dificuldade, e sim, em nível de abstração, tendo em sua base as experiências mais concretas e em direção ao topo as experiências mais abstratas. Também é importante notar que uma experiência não ocorre necessariamente isolada das demais e tampouco transitam sequencialmente de uma para a outra.

Esta imagem destaca que o método tradicional de ensino prioriza as experiências de aprendizagem da parte superior do cone, enquanto que as metodologias ativas aplicadas à educação também valorizam as experiências próximas à base do cone de aprendizagem.

Fica, portanto, evidente perceber que apenas o ensino tradicional não é suficientemente capaz de suprir com todas as demandas para uma formação mais completa e integrada, a fim de promover o desenvolvimento das inúmeras competências desejáveis para auxiliar os alunos a enfrentarem os desafios de cunho social, político, econômico e ambiental do século XXI.

\section{ALGUNS CASOS DE APRENDIZAGEM ATIVA EM CURSOS DE ENGENHARIA}

Na sequência são apresentados dois casos de aplicação da aprendizagem ativa, sob a perspectiva dos estudantes, em disciplinas dos cursos de engenharia na Escola Politécnica da USP e na Escola Politécnica da UFRJ. O objetivo é mostrar como múltiplas competências são abordadas pela aprendizagem ativa na formação de engenheiros e que tais práticas já estavam 
previamente alinhadas às atuais DCNs de Engenharia. Assim, o sucesso de tais atividades corroboram para a aplicação da aprendizagem ativa como metodologia de ensino.

\subsection{Aprendizagem baseada em projetos na disciplina Instrumentação e Métodos Experimentais em Mecânica dos Fluidos da Escola Politécnica da UFRJ}

Esta disciplina é regularmente oferecida pelo Departamento de Engenharia Mecânica da Escola Politécnica da UFRJ, sendo parte do currículo do curso de graduação em engenharia mecânica. A disciplina tem como principal objetivo o desenvolvimento de competências relacionadas ao trabalho experimental em engenharia com foco na mecânica dos fluidos. Para isso, adotou-se como principal estratégia pedagógica o aprendizado baseado em projetos.

Detalha-se aqui o oferecimento da disciplina referente ao ano de 2016, que contou com 10 estudantes do $7^{\circ}$ período de engenharia mecânica, tendo sido cursada por um dos autores do presente trabalho.

Na primeira aula, os estudantes foram organizados em grupos de até 5 alunos. As equipes formadas permaneceram as mesmas no decorrer da disciplina, tanto para a realização das atividades práticas dirigidas como para o desenvolvimento do projeto de conclusão.

A disciplina foi dividida em duas etapas, sendo estas:

- Aulas expositivas e práticas dirigidas: etapa caracterizada pela exposição do conteúdo teórico com utilização de recursos audiovisuais e quadro branco seguida de realização de atividades práticas dirigidas com supervisão do docente. Esta etapa foi realizada durante seis aulas, nas primeiras semanas letivas do semestre. A exposição do conteúdo era realizada no momento inicial da aula, utilizando, em média, trinta minutos. O tempo restante era utilizado no desenvolvimento das atividades práticas previamente planejadas pela professora. Eventualmente, durante as atividades práticas, a professora utilizava alguns momentos para destacar, questionar ou esclarecer aspectos teóricos.

- Desenvolvimento do projeto: etapa caracterizada pela total autonomia dos grupos no desenvolvimento dos seus projetos sob orientação da docente da disciplina.

Os projetos foram construídos, testados e calibrados no laboratório. Os dados adquiridos nos diversos testes realizados e no processo de calibragem foram tratados estatisticamente e incluídos no relatório final. Os relatórios foram elaborados no decorrer da segunda etapa da disciplina sob orientação do docente responsável.

A avaliação de desempenho dos estudantes foi realizada utilizando dois parâmetros: a observação do envolvimento intelectual dos estudantes no desenvolvimento das atividades e a análise final do projeto desenvolvido (protótipo e relatório final). A principal meta da disciplina foi o desenvolvimento de um protótipo funcional e a elaboração de um relatório.

A disciplina envolveu a aplicação de uma série de conteúdos estudados ao longo do curso de engenharia, estimulando também o estudo de tópicos de eletrônica (não cobertos por disciplinas do curso). Além disso, os estudantes tiveram a oportunidade de pôr a mão na massa, enfrentando os desafios dos problemas reais de engenharia e adquirindo experiência no desenvolvimento de projetos em equipe.

\subsection{Aprendizagem ativa na disciplina Introdução à Engenharia na Escola Politécnica da USP - uma análise do oferecimento de 2013}

A disciplina Introdução à Engenharia propõe o desenvolvimento de um projeto de engenharia a fim de que os estudantes possam compreender e aplicar o método de engenharia. Os ingressantes da Escola Politécnica são divididos em 24 turmas de 32 alunos. Em cada turma, são formados oito grupos com quatro estudantes. As turmas são constituídas por 
estudantes de diferentes cursos com a finalidade de formar grupos interdisciplinares de trabalho.

Neste texto, é detalhado o oferecimento da disciplina referente ao ano de 2013, onde a temática para o desenvolvimento dos projetos foi "meios de transporte sustentáveis". O projeto foi iniciado com a identificação das necessidades dos usuários em cada tipo dos meios de transporte e formulação das primeiras alternativas de solução. Na sequência, houve competição entre os grupos, de forma a dar espaço para o julgamento da turma sobre qual dos projetos havia apresentado um trabalho mais completo, estimulando os estudantes no desenvolvimento de habilidades de comunicação. Após essa etapa, os integrantes dos grupos eram substituídos, permanecendo apenas um ou dois elementos originais em cada grupo para preservar a essência da ideia que estava sendo desenvolvida. Os novos integrantes verificavam o trabalho produzido pelo grupo até aquele momento e davam continuidade, avaliando e realizando a seleção das alternativas. Ao final, cada grupo deveria escolher uma das soluções ou propor uma integração de soluções complementares. Novamente, os grupos apresentavam os resultados para a turma e era realizada uma votação sobre o desempenho dos grupos. De cada turma, o projeto com os melhores resultados obtidos era selecionado para uma nova competição entre diferentes turmas, estimulando a competitividade entre os projetos.

Com o envolvimento de uma série de conhecimentos de diferentes áreas, a disciplina possibilitou que os estudantes tivessem a oportunidade de dar os primeiros passos na aplicação do método de engenharia na busca de solução para um problema atual da sociedade. Além disso, a disciplina destacou a importância do trabalho em equipe e do desenvolvimento de habilidades de comunicação no exercício da engenharia.

\section{CONSIDERAÇÕES FINAIS}

No processo de transformação digital dos setores produtivos, a aprendizagem ativa possui um papel estratégico na formação de profissionais de engenharia preparados para lidar com os desafios da economia atual. O desenvolvimento de múltiplas competências é fundamental na formação de engenheiros capazes de produzir conhecimento, desenvolver tecnologias e inovações. Neste sentido, as novas Diretrizes Curriculares Nacionais do Curso de Graduação em Engenharia, instituídas em 2019, destacam a importância da implementação de metodologias de ensino baseadas na aprendizagem ativa com intuito de promover uma educação centrada no estudante e preocupada com o desenvolvimento de competências.

No entanto, a indicação da aprendizagem ativa como estratégia didática é apenas um dos passos para a sua implementação. É preciso organizar um processo de formação de professores para que estes compreendam profundamente seus princípios, percebam o seu potencial educacional e sintam-se apoiados e encorajados pelas instituições de ensino a atualizar suas práticas educacionais.

Mudanças em processos, em especial nos de longa data como o educacional, são sempre difíceis, seja pelo comodismo ou temor de insucessos futuros. Contudo, aqui, foram apresentados casos reais de aplicação da aprendizagem ativa que indicam que é possível adotar tal prática com sucesso, desde que bem planejada pelo professor.

Espera-se que este seja mais um trabalho que possa contribuir efetivamente para a adoção da aprendizagem ativa nos cursos de engenharia e de outras graduações das universidades brasileiras. 
"Os desafios para formar hoje o engenheiro do amanhã"

\section{Agradecimentos}

Ao apoio da Coordenação de Aperfeiçoamento de Pessoal de Nível Superior - Brasil (CAPES) - Código de Financiamento 001, para a realização do presente trabalho.

\section{REFERÊNCIAS}

ACLAND, Charles Reid. American AV: Edgar Dale and the Information Age Classroom. Technology and Culture, v. 58, n. 2, p. 392-421, 2017. DOI: 10.1353/tech.2017.0041.

ANDERSON, Lorin W. et. al. A taxonomy for learning, teaching and assessing: a revison of Bloom's Taxonomy of Educational Objectives. Nova York: Addison Wesley Longman, 2001. 336 p.

BERBEL, Neusi Aparecida Navas. As metodologias ativas e a promoção da autonomia de estudantes. Semina: Ciências Sociais e Humanas, v. 32, n. 1, p. 25-40, 2011.

CASTANHA, André Paulo. Os métodos de ensino no Brasil do século XIX. Revista HISTEDBR On-line, v. 17, n. 4, p. 1054-1077, 21 dez. 2017. DOI: 10.20396/rho.v17 i4.8651232.

DA SILVA, Fábio Luiz; MUZARDO, Fabiane Tais. Pirâmides e cones de aprendizagem: da abstração à hierarquização de estratégias de aprendizagem. Dialogia, n. 29, p. 169-179, 2018. DOI: 10.5585/dialogia.N29.7883.

DALE, Edgar. Audiovisual methods in teaching. 3.ed. New York: Dryden Press,1954. $534 \mathrm{p}$.

FREIRE, Paulo. Pedagogia do Oprimido. Rio de Janeiro, RJ: Paz e Terra, 268 p., 1987.

Global Innovation Index. Disponível em: 〈https://www.globalinnovationindex.org/Home> . Acesso em: 24 jul. 2020.

GONÇALVES, Andreza; SCAICO, Pasqueline. O Desafio de Professores da Licenciatura em Computação ao Usarem Práticas de Aprendizagem Ativa: Um Estudo de Caso na Universidade Federal da Paraíba. In: Anais dos Workshops do Congresso Brasileiro de Informática na Educação, v. 8, n. 1, 2019. p. 91. DOI: 10.5753/cbie.wcbie.2019.91.

HARKINS, Arthur M. et al. Leapfrog principles and practices: Core components of education 3.0 and 4.0. Futures Research Quarterly, v. 24, n. 1, p. 19-31, 2008.

INSTRUCTION at FSU: A Guide to Teaching and Learning Practices. Center for Teaching \& Learning, 6th edition, Florida State University, 2010.

MARIN, Maria José Sanches et al. Aspectos das fortalezas e fragilidades no uso das metodologias ativas de aprendizagem. Revista brasileira de educação médica, v. 34, n. 1, p. 13-20, 2010. DOI: 10.1590/S0100-55022010000100003. 
Ministério da Educação (MEC). Relatório de elaboração das Diretrizes Curriculares Nacionais do Curso de Graduação em Engenharia. Disponível em: <http://portal.mec .gov.br/docman/marco-2019-pdf/109871-pces001-19-1/file>. Acesso em: 24 jul. 2020.

MORÁN, José. Mudando a educação com metodologias ativas. Coleção mídias contemporâneas. Convergências midiáticas, educação e cidadania: aproximações jovens, v. 2, n. 1, p. 15-33, 2015.

MORÁN, José. Como Transformar Nossas Escolas. In CARVALHO, Mônica Timm de. (Org). Educação 3.0 - Novas Perspectivas para o Ensino. São Leopoldo, RS: Editora Unisinos. pp. 63-87 2017.

SAVIANI, Dermeval et al. O legado educacional do século XIX. Campinas, SP: Autores Associados, 2017.

SILVEIRA, Marcos Azevedo da. Formação do Engenheiro Inovador: uma visão internacional. Sistema Maxwell, PUC, Rio de Janeiro, 2005.

World Intellectual Property Organization (WIPO). The Global Innovation Index Report. 2019.

WORLD ECONOMIC FORUM (WEF). Readiness for the future production Report. 2018.

\title{
ACTIVE LEARNING AS THE MAIN STRATEGY TO COMPLY WITH THE NEW NATIONAL CURRICULAR GUIDELINES IN ENGINEERING
}

\begin{abstract}
The engineering education in Brazil faces great challenges during the digital transformation of the global economy and the so-called "universalization of knowledge". The curricular guidelines have been improved graduate professionals capable of promoting the development of technologies, innovation and producing knowledge, tasks that are closely linked to the engineering profession. In this context, a wide discussion has been carried out about active learning methods, which aim to bring the student to the center of the learning process, motivating him to seek knowledge by himself. For this to occur, there are necessary institutional actions at the university and a change in the understanding of the professors and students roles, to enhance a more structuring learning. This paper presents a review of active learning strategies in engineering courses in the light of the new National Curriculum Guidelines for the Undergraduate Engineering Course. It also discusses the effectiveness of these strategies facing current challenges in engineering education and their advantages and disadvantages compared to the traditional teaching method. Finally, two practical cases of applying active learning in engineering courses are analyzed and discussed.
\end{abstract}

Keywords: Active Learning. Engineering Learning. Engineering National Curriculum Guidelines. Learning Pyramid. 